\title{
Tuberculous liver abscess; a rare manifestation of extra-pulmonary tuberculosis
}

\author{
Aravinthan M, Kodikara Arachchi RW \\ Teaching Hospital, Karapitiya, Galle \\ Corresponding author;Dr.M.Aravinthan (ara.jhc@gmail.com)
}

\section{Introduction}

Liver involvement has been reported in $10-15 \%$ of patients with pulmonary tuberculosis and it is a common finding in patients with disseminated tuberculosis (1). Tuberculous liver abscess (TLA) is extremely rare even in countries where tuberculosis is an alarming public health problem. It is usually associated with foci of infection either in lung and or gastrointestinal tract in an immuno-compromised state (2). The prevalence of TLA was $0.34 \%$ in patients with hepatic tuberculosis as shown in a study involving patients between 6 months to 72 years of age (3). An isolated primary TLA with no evidence of tuberculosis elsewhere is even rarer. The diagnosis is difficult and it mimics hepatoma, pyogenic liver abscess or amoebic liver abscess $(4,5)$.

\section{Case Report}

A 59-year-old trader with alcoholic liver disease was admitted with intermittent mild fever, anorexia, abdominal discomfort following meals, of one month duration. He also had mild, intermittent right upper abdominal pain of the same duration. There was no history of cough, recent blood transfusions, loss of weight, contact with a tuberculosis patient, history of illicit drug intake, foreign travel or extra marital sexual relationships. On examination, mild pallor, mild icterus, bilateral parotid gland enlargement and mild hepatosplenomegaly were noted. Examination of all the other systems was unremarkable. Investigations showed haemoglobin $7.5 \mathrm{~g} / \mathrm{dL}, \mathrm{WBC} 8.2 \times 10^{9} / \mathrm{L}$ and platelets $256 \times 10^{9} / \mathrm{L}$. Blood picture was compatible with cold type auto immune haemolytic anaemia. ESR was $97 \mathrm{~mm}$ in the first hour and CRP was $6.4 \mathrm{mg} / \mathrm{dL}$. Direct and indirect Coomb's tests were positive. Bone marrow aspiration showed erythroid hyperplasia. Serum total bilirubin was $30 \mathrm{~g} / \mathrm{L}$ and indirect bilirubin was 18 $\mathrm{g} / \mathrm{L}$. Repeated blood cultures were negative.
Hepatitis B surface antigen, VDRL and HIV antibodies were negative. Liver transaminases and serum creatinine were normal. Chest radiograph and ECG showed no abnormalities. Sputum AFB (three times) and Mantoux test were negative. Abdominal ultrasonography showed two cystic lesions $(2.6 \mathrm{~cm}$ $3.9 \mathrm{~cm}$ ) in the right lobe of liver and mild hepatosplenomegaly. CT abdomen suggested the possibility of multifocal hepatoma or multiple liver abscesses (Figure 1). Ultra sound guided aspiration of suspected lesions showed protein $12 \mathrm{~g} / \mathrm{dL}$, pus cells $3+$. Direct smear of the liver aspirate revealed acid fast bacilli and the culture was positive for Mycobacterium tuberculosis (Figure 2).

He was treated with 'SHE' regimen (Streptomycin 1 $\mathrm{g} / \mathrm{d}$, INAH $300 \mathrm{mg} / \mathrm{d}$, Ethambutol $900 \mathrm{mg} / \mathrm{d}$ ) in the first two months. After that 'HE 'regimen (INAH and Ethambutol same doses) was started and continued. After two months of anti-tuberculous treatment, drugs were temporary withdrawn for a week due to worsening of transaminases and serum bilirubin. Drugs were restarted once these tests became normal. Patient responded to anti-tuberculous treatment and after six months of treatment, repeat ultrasound scan showed complete resolution of liver cystic lesions.

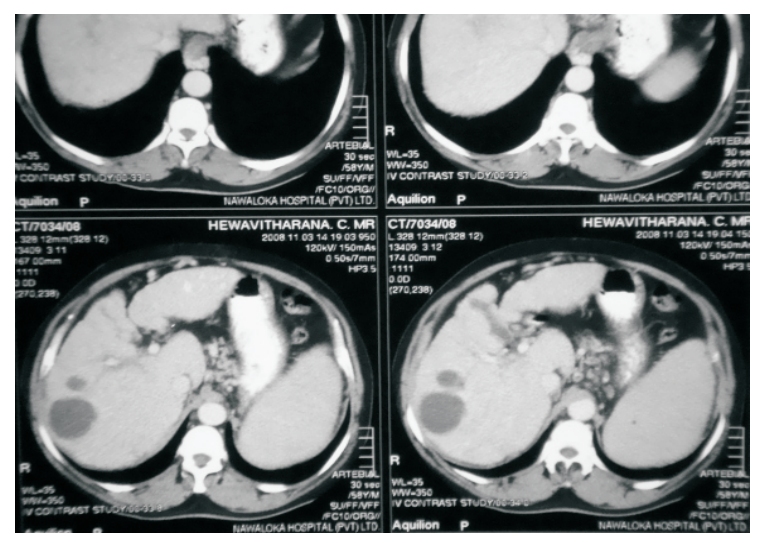

Figure 1 - CT Abdomen 


\section{Discussion}

TLA was first described by Beslowe in 1858 (6). Approximately 100 cases of TLAs have been described in the literature (7). Three forms of tuberculous liver involvement are described. They are diffuse involvement associated with miliary or pulmonary tuberculosis, diffuse parenchymal involvement without any evidence of existing tuberculosis elsewhere (primary miliary tuberculosis of liver), and focal or nodular lesions in the liver which may be multiple or solitary and present as tuberculoma or abscess (8).

Clinical manifestations are frequently non-specific and include weight loss, anorexia, fever, abdominal pain and jaundice. Hepatomegaly, right-sided hypochondrial pain and icterus are usual clinical findings (8). Diagnosis is confirmed by the demonstration of AFB in direct smear of aspirated pus or in biopsy of abscess wall. Indirect evidence like ELISA / PCR may be helpful if one fails to demonstrate the AFB. AFB on smear has sensitivity of $0-45 \%$ while PCR has $57 \%$ sensitivity and TB culture $10-60 \%$.

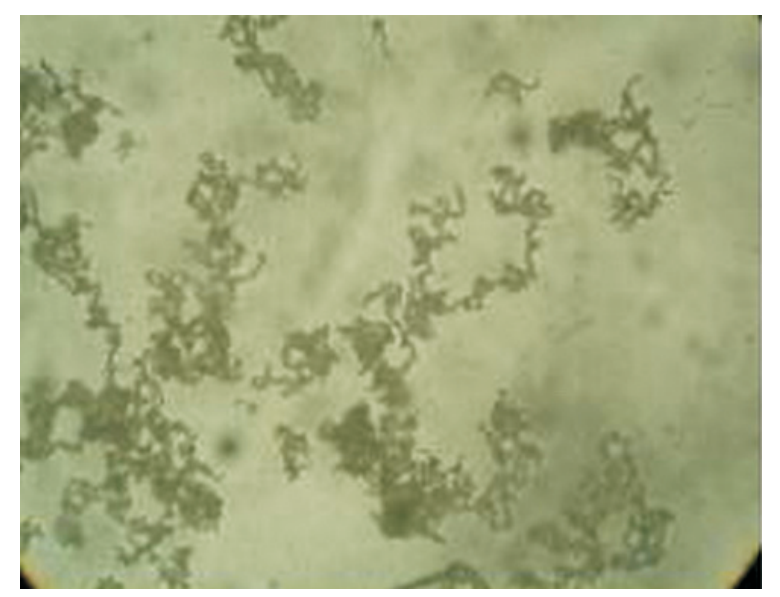

Figure 2 - TB culture (Liver abscess)

Quadruple therapy is recommended generally for one year (9). Gracey postulates that thick fibrous tissue around the abscess and their large size may prevent antibiotics from reaching the target (10). Mustard et al treated tuberculous liver abscess by local infusion of anti tuberculous drugs. Surgery is reserved for multiple large lesions and lesions refractory to medical treatment (11). Prognosis of TLA is good for the majority of patients if diagnosed early and prompt therapy is started (9).
Anaemia is present in $63 \%$ of miliary TB patients. Several mechanisms have been proposed such as immune, non-immune mechanisms, nutritional deficiency, failure of iron utilization, malabsorption, marrow suppression and haemolysis. Disappearance of haematological abnormalities following anti TB drugs alone is an adequate proof that $\mathrm{TB}$ is the underlying cause of haemolytic anaemia. Recovery of $\mathrm{Hb}$, reticulocyte, haptoglobin, bilirubin, Coombs negativity following anti TB treatment are taken as supportive evidence of immune-mediated haemolytic anaemia due to tuberculosis aetiology. The association of auto immune haemolytic anaemia with tuberculosis, although extremely rare, is known for quiet long. To our knowledge since then seven cases of auto immune haemolytic anaemia in association with tuberculosis have been reported in medical literature (12).

In our patient, clinical symptoms improved with anti tuberculous treatment. Repeat abdominal ultrasonography after six months of anti tuberculous treatment showed the disappearance of liver cystic lesions. We intend to repeat Coombs test and other investigations after the completion of anti tuberculous treatment to prove the TB aetiology

\section{References}

1. Nilyanand, Agarwal HK, Singh Manmect. Tuberculous liver abscess. Journal of the Association of Physicians, India 2000; 48: 244.

2. Akcay MN, Polat KY, Oren D, Ozturna. Primary tuberculous liver abscess:a case report and review of literature. International Journal of Clinical Practice 2004; 58(6): 625-7.

3. Essop AR, Segal I, Posen J, Noor Mohamed N. Tuberculous abscess of liver. South African Medical Journal 1983; 63: 825-6.

4. Balsakar DD, Joshi MA. Isolated tuberculosis hepatic abscess in a non-immunocompromised patient. Journal of Postgraduate Medicine 2000; 46(2): 108-9.

5. Roy R, Goyal RK, Gupta N. Tuberculous liver abscess-a case report. Journal of Indian Association of Physicians 2000; 48: 241.

6. Gracey L. Tuberculous abscess of liver. British Journal of Surgery $1965 ; \mathbf{5 2}: 442-4$. 
7. Reed DH, Nash AF, Valabhji P. Radiological diagnosis and management of a solitary tuberculous hepatic abscess. British Journal of Radiology 1990; 63: 902-4.

8. Stevens A, Little JM. Isolated tuberculous hepatic abscess. Aust NZ Journal of Surgery. 1987; 57: 409-11.

9. Parsak CK, Hanta I, Aslan A, Alabaz O. Case reports in Gastroenterology 2008; 2: 18-21.
10. Nampory MR, Halim MM, Shreedharan R, Al sweih NA, Gupta RK, Costandi JN. Liver abscess and DIC in tuberculosis. Postgraduate Medical Journal 1995; 71 : $490-2$.

11. Mustard RA, Mackenzie RL, Gray RG. Percutaneous drainage of a tuberculous liver abscess. Canadian Journal of Surgery 1986; 29: 449-50.

12. Abbas Z, Cunningham R, Wilkinson SP. Journal of Infection 2000; 40(3): 291-2.

\title{
Non-Hodgkins lymphoma of the thyroid gland; a case report
}

\author{
Banagala ASK, Liyanage AS \\ Colombo South Teaching Hospital, Kalubowila, Dehiwala \\ Corresponding author;Dr.A.S.K.Banagala (nurabsurg@gmail.com)
}

\section{Case Report}

A 56-year-old female was admitted to the medical casualty ward with stridor. Examination revealed a moderate sized goiter and her trachea could not be palpated. Her lungs were clinically normal and the radiographs showed a marked tracheal shift to the right side with a narrow subglottic airway (Fig 1,2). The patient also complained of dysphagia and hoarseness of voice. A nasogastric tube was inserted and oxygen inhalation was started with a venti mask. Intravenous steroids too were given. CT scan of the neck and chest revealed a large left thyroid growth with a few enlarged lymph nodes in the superior mediastinum (Fig 3). The thyroid growth was seen to extend posterior to the trachea and larynx, and retrosternally too. Indirect laryngoscopy revealed left vocal cord palsy. Fine needle aspiration cytology showed malignant cells.

After aneasthetic and cardiac risk assessment, elective thyroidectomy was planned. All attempts at endotracheal intubation including direct laryngoscopy and guided intubation, failed. An elective tracheostomy followed by left hemithyroidectomy was performed. Left recurrent laryngeal nerve could not be identified. The left thyroid lobe was dissected off the left carotid vessels and the left internal jugular vein. The right thyroid lobe appeared normal. Postoperatively, the patient was ventilated electively for 48 hours. After an apparent initial clinical improvement the patient developed swelling of both upper limbs and the head/neck region indicating obstruction of the superior vena cava. Meanwhile, the histology of the resected thyroid confirmed a non-Hodgkins lymphoma (NHL). Three cycles of combination chemotherapy and systemic steroids did not help and the patient died on the post-op day 21.

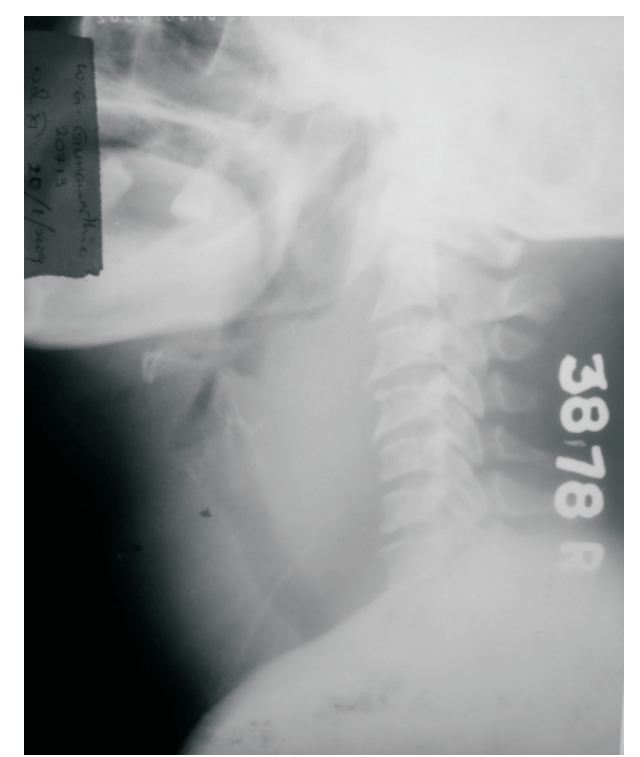

Figure 1 - Lateral radiograph of the neck 